DIVISION OF THE HUMANITIES AND SOCIAL SCIENCES

CALIFORNIA INSTITUTE OF TECHNOLOGY

PASADENA, CALIFORNIA 91125

INEQUALITY AVERSION AND RISK AVERSION

Christopher P. Chambers

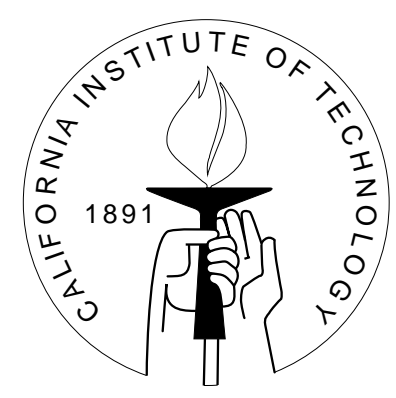

SOCIAL SCIENCE WORKING PAPER 1300

March 2009 


\title{
Inequality aversion and risk aversion
}

\author{
Christopher P. Chambers*
}

\begin{abstract}
This note shows that for two social welfare functions which are inequality averse and anonymous, if one is more inequality averse than the other, it induces a more risk averse household through optimal sharing than the other. We present examples showing that this comparative static can be reversed if either the property of inequality aversion or anonymity is dropped.
\end{abstract}

\section{Introduction}

The result in this note studies the interconnection between inequality aversion, on the one hand, and risk aversion, on the other. A common observation is that inequality aversion and risk aversion often go hand-in-hand. For instance, a risk averse decision maker choosing among income distributions (not knowing in which part of the distribution he will reside) is indistinguishable from an inequality averse social planner.

In this work, we tackle the interplay between inequality aversion and risk aversion from a different perspective. A household of individuals shares risk in order to optimize some social welfare function; the individuals may differ in terms of their risk attitudes, but hold common beliefs. The risk comes in aggregate form and must be distributed amongst the agents. We follow Samuelson (1956) and Chipman and Moore (1979) in studying household behavior under various social welfare functions. Social welfare functions can be ranked according to their propensity to reduce inequality. A simple intuition would suggest that the more inequality averse a social welfare function, the more it must reflect all agents preferences. The more it reflects all individuals preferences, in particular, the more it reflects the "weakest" or most risk-averse individual's preferences. Less inequality averse social welfare functions would not seem to have this propertymaximizing aggregate utility for example would always allocate risk to the agents who benefit the most from it; these are the least risk averse agents. Using this naive intuition, we could conclude that more inequality averse households would tend to be more risk averse.

*This note grew out of discussions with Federico Echenique and Mark Machina, toward whom I am grateful. 
This intuition turns out to be true up to a point, and this is our main result. This simple comparative static holds for a broad family of social choice rules: namely, those which are anonymous (social utility depends only on the distribution of utilities) and inequality averse in an absolute sense: reducing inequality always benefits society. A plethora of rules exhibit these two properties; indeed, any social welfare function which is symmetric and quasiconcave has them. For this family of rules, a more inequality averse social welfare function induces a more risk averse household preference.

However, this intuition breaks down upon removing one of the two properties. It turns out the critical feature of anonymous and inequality averse social welfare functions is that equal division is always a socially optimal allocation of a certain dollar. Equal division of a dollar always results in equal utilities. For an aggregate risky prospect to be preferred to a certain dollar, it must therefore be the case that there is an allocation of that risky prospect which is socially better than equal division of that dollar. And if this is true for a social welfare function, it is certainly also true for a less inequality averse social welfare function.

When dropping either of the two properties, however, equal division of a certain dollar need not be socially optimal. In general then a comparison of the induced preferences given two social welfare functions ranked in terms of inequality aversion need not exist. The key feature of an equal division of a dollar is that it results in an equal distribution of utilities: the property of inequality aversion is about how a list of utilities compares to equal division. Without the equal division benchmark, it turns out that anything can happen.

Section 2 illustrates the issues involved with a simple example. Section 3 provides the main result of the paper. Section 4 provides examples illustrating how the conclusion of the theorem can break down when assumptions are dropped. Finally, Section 5 concludes.

\section{Three social welfare functions}

There are two individuals living in a household, Alice and Bob. The household faces aggregate uncertainty about a monetary payoff, which depends on the state of nature, Rain or Shine $(R$ or $S)$. Each individual has a quasiconcave utility function, which is homogeneous of degree one, over risky consumption. For example, Alice's utility for $\left(x_{R}, x_{S}\right)$ is $U_{A}\left(x_{R}, x_{S}\right)$. Utilities are further assumed to be measured in dollars, so that for a constant consumption bundle $(c, c), U_{i}(c, c)=c$. Lastly, the utilities have a common prior $\left(\pi_{R}, \pi_{S}\right)$, which is simply a vector for which for all certain consumption $(c, c)$,

$$
U_{i}\left(x_{R}, x_{S}\right) \geq c \text { implies } \pi_{R} x_{R}+\pi_{S} x_{S} \geq c .
$$

The normalization of utility reflects the fact that utility is measured in dollar amounts-the utility of risky consumption is equal to its certainty equivalent. This facilitates comparison of utilities across agents (money is a natural metric). 
Alice and Bob must decide how to rank aggregate consumption. They decide to allocate aggregate consumption according to a social choice rule. The social choice rule maps individual utilities into an aggregate utility: $W: \mathbb{R}_{+}^{2} \rightarrow \mathbb{R}$. The specification of this choice rule determines the ranking over aggregate consumption. Three social choice rules, which take as input utility, are as follows:

i) The maximax rule:

$$
W_{\max }\left(u_{1}, u_{2}\right)=\max \left\{u_{1}, u_{2}\right\}
$$

ii) The utilitarian rule:

$$
W_{U}\left(u_{1}, u_{2}\right)=u_{1}+u_{2}
$$

and

iii) The maximin (Rawlsian) rule

$$
W_{\min }\left(u_{1}, u_{2}\right)=\min \left\{u_{1}, u_{2}\right\} .
$$

The three rules differ as to their inequality aversion. The maximax rule is, in a sense, inequality loving, while the utilitarian is inequality neutral, and the maximin is inequality averse. We ask how the behavior of the rules in terms of inequality aversion affects the household's behavior in terms of risk aversion. A simple intuition is that, the more inequality averse the social welfare function, the more risk averse the induced household preference. We show that this intuition breaks down in a strong way in this example.

The household's utility of an aggregate bundle $\left(x_{R}, x_{S}\right)$ is given by

$$
\max _{\substack{x_{R}^{A}+x_{R}^{B}=x_{R} \\ x_{S}^{A}+x_{S}^{B}=x_{S}}} W\left(U_{A}\left(x_{R}^{A}, x_{S}^{A}\right), U_{B}\left(x_{R}^{B}, x_{S}^{B}\right)\right) .
$$

The household utility therefore depends on the social welfare function under consideration. We can ask how these household utilities fare as to risk aversion. One way to verify this, using a classical characterization of risk aversion, is to compute the at least as good as sets for a constant aggregate bundle $(c, c)$ (these are the set of aggregate bundles that the household ranks at least as good as a sure thing paying out $c$ ).

Denote the individual at least as good as sets for $(c, c)$ for $i=A, B$ by

$$
\bar{U}^{i}(c, c)=\left\{\left(x_{R}, x_{S}\right): U_{i}\left(x_{R}, x_{S}\right) \geq U_{i}(c, c)=c\right\}
$$

It is a straightforward exercise to compute the at least as good as sets for the household, given each of the social choice rules introduced above. For the maximax rule, the at least as good as set for $(c, c)$ is

$$
\bar{U}^{\max }(c, c)=\bar{U}^{A}(c, c) \cup \bar{U}^{B}(c, c) .
$$


For the utilitarian rule, it is

$$
\bar{U}^{U}(c, c)=\operatorname{co}\left(\bar{U}^{A}(c, c) \cup \bar{U}^{B}(c, c)\right) .
$$

For the maximin rule, it is

$$
\bar{U}^{\min }(c, c)=\frac{1}{2} \bar{U}^{A}(c, c)+\frac{1}{2} \bar{U}^{B}(c, c) .
$$

Therefore, for this example, $\bar{U}^{\max }(c, c) \subset \bar{U}^{U}(c, c)$ and $\bar{U}^{\min }(c, c) \subset \bar{U}^{U}(c, c)$, with strict inclusions (in general). In general there is no relation between $\bar{U}^{\text {max }}(c, c)$ and $\bar{U}^{\text {min }}(c, c)$. These two set inclusions demonstrate that the utilitarian social welfare function leads to a less risk averse household than either the maximax or the maximin planner. This coincides with our intuition for the maximin case, but not for the maximax case.

\section{The explanation}

The intuition that greater inequality aversion leads to greater risk aversion turns out to be true, but only with some caveats. The comparative static does not hold for all pairs of social welfare functions. First, note that, in the example, while the maximax social welfare function is less inequality averse than the utilitarian social welfare function, it is not, in an absolute sense, inequality averse. This fact turns out be responsible for the breakdown in our intuition.

Secondly, note that all three social welfare functions in the example are anonymous; that is, $W\left(u_{A}, u_{B}\right)=W\left(u_{B}, u_{A}\right)$. Social welfare functions violating this property will also falsify the comparative static in general.

Let us let $N=\{1, \ldots, n\}$ denote a finite household of agents, and $\Omega=\{1, \ldots, \omega\}$ a finite set of states. Each individual has a utility function $U_{i}: \mathbb{R}_{+}^{\Omega} \rightarrow \mathbb{R}$ which is increasing, continuous, and quasiconcave; we further assume as in the example that utility functions are normalized so that for any constant bundle $(c, c, \ldots, c), U_{i}(c, \ldots, c)=c$. As in the example, this is a natural metric for comparing utility across agents. We lastly assume, as in the example, that there is a common prior $\left(\pi_{1}, \ldots, \pi_{\omega}\right)$ for which for all $c$,

$$
U_{i}\left(x_{1}, \ldots, x_{\omega}\right) \geq c \Longrightarrow \sum_{s=1}^{\omega} \pi_{s} x_{s} \geq c .
$$

A social welfare function $W: \mathbb{R}_{+}^{N} \rightarrow \mathbb{R}$ is inequality averse if it is quasiconcave; it is anonymous if for any relabelling of the names of agents, the social utility does not change. We assume all social welfare functions are monotonic and continuous. For two social welfare functions $W, W^{\prime}$, we will say that $W$ is more inequality averse than $W^{\prime}$ if for any $\left(u_{1}, \ldots, u_{n}\right)$ and $(v, v, \ldots, v)$,

$$
W\left(u_{1}, \ldots, u_{n}\right) \geq W(v, \ldots, v) \Longrightarrow W^{\prime}\left(u_{1}, \ldots, u_{n}\right) \geq W^{\prime}(v, \ldots, v) .
$$


That is, any deviation from equality of utility which $W$ finds acceptable, $W^{\prime}$ finds acceptable as well. ${ }^{1}$

The social welfare function generates a household utility function, given by

$$
U_{W}\left(x_{1}, \ldots, x_{\omega}\right)=\max _{\sum_{i=1}^{n} x_{s}^{i}=x_{s}} W\left(U_{1}\left(x_{1}^{1}, \ldots, x_{\omega}^{1}\right), \ldots, U_{n}\left(x_{1}^{n}, \ldots, x_{\omega}^{n}\right)\right)
$$

That is, the household utility is the maximal social utility that can be achieved with an aggregate bundle.

We now say that household utility $U_{W}$ is more risk averse than household utility $U_{W^{\prime}}$ if for any constant aggregate bundle $(c, c, \ldots, c)$ and any $\left(x_{1}, \ldots, x_{\omega}\right)$,

$$
U_{W}\left(x_{1}, \ldots, x_{\omega}\right) \geq U_{W}(c, c, \ldots, c) \Longrightarrow U_{W^{\prime}}\left(x_{1}, \ldots, x_{\omega}\right) \geq U_{W^{\prime}}(c, c, \ldots, c) .
$$

That is, any deviation from uncertainty which $U_{W}$ finds acceptable, $U_{W^{\prime}}$ also finds acceptable. $^{2}$ Note the parallel with the comparative notion of inequality aversion. This parallel is only superficial: with inequality aversion, $W$ ranks $N$-vectors, whereas with risk aversion, $U_{W}$ ranks $\Omega$-vectors.

This brings us to the main result:

Theorem 1 Suppose that $W$ and $W^{\prime}$ are anonymous and inequality averse social welfare functions. Then if $W$ is more inequality averse than $W^{\prime}, U_{W}$ is more risk averse than $U_{W^{\prime}}$.

The proof of the theorem is extremely simple and is illustrated in Figure 1. A critical step in the proof shows that for any constant bundle $(c, \ldots, c)$, the utility possibility set is a simplex. Denote the utility possibility set of $\left(x_{1}, \ldots, x_{\omega}\right)$ by $\mathcal{U}(x)$. Supposing that $U_{W}\left(x_{1}, \ldots, x_{\omega}\right) \geq U_{W}(c, \ldots, c)$ means there is a point on $\mathcal{U}(x)$ which $W$ ranks as at least as high as every point on the $c$-simplex. But as the maximizers of $W$ and $W^{\prime}$ on this $c$ simplex coincide and are equal to the ray of equal coordinates, since $W$ is more inequality averse than $W^{\prime}$, the relevant upper contour set of $W$ is contained in the upper contour set of $W^{\prime}$. Therefore, there is a point on $\mathcal{U}(x)$ which $W^{\prime}$ ranks at least as high as every point on the $c$-simplex.

Proof: Let $(c, c, \ldots, c) \in \mathbb{R}_{+}^{\Omega}$. We claim that for both $W$ and $W^{\prime}$, a maximizer to the function

$$
\max W\left(U_{1}\left(x_{1}^{1}, \ldots, x_{\omega}^{1}\right), \ldots, U_{n}\left(x_{1}^{n}, \ldots, x_{\omega}^{n}\right)\right)
$$

subject to $\sum_{i=1}^{n} x_{s}^{i}=c$ is given by $x_{s}^{i}=\frac{c}{n}$ for all $i \in N, s \in \Omega$. To see this, note that all allocations $\left\{\left(x_{1}^{1}, \ldots, x_{\omega}^{1}\right), \ldots,\left(x_{1}^{n}, \ldots, x_{\omega}^{n}\right)\right\}$ of $(c, c, \ldots, c)$ are weakly Pareto dominated by

\footnotetext{
${ }^{1}$ The comparative notion of inequality aversion is essentially due to Atkinson (1970). It parallels the comparative notion of risk aversion pioneered by Yaari (1969).

${ }^{2}$ As noted, this general comparative notion of risk aversion is due to Yaari (1969).
} 


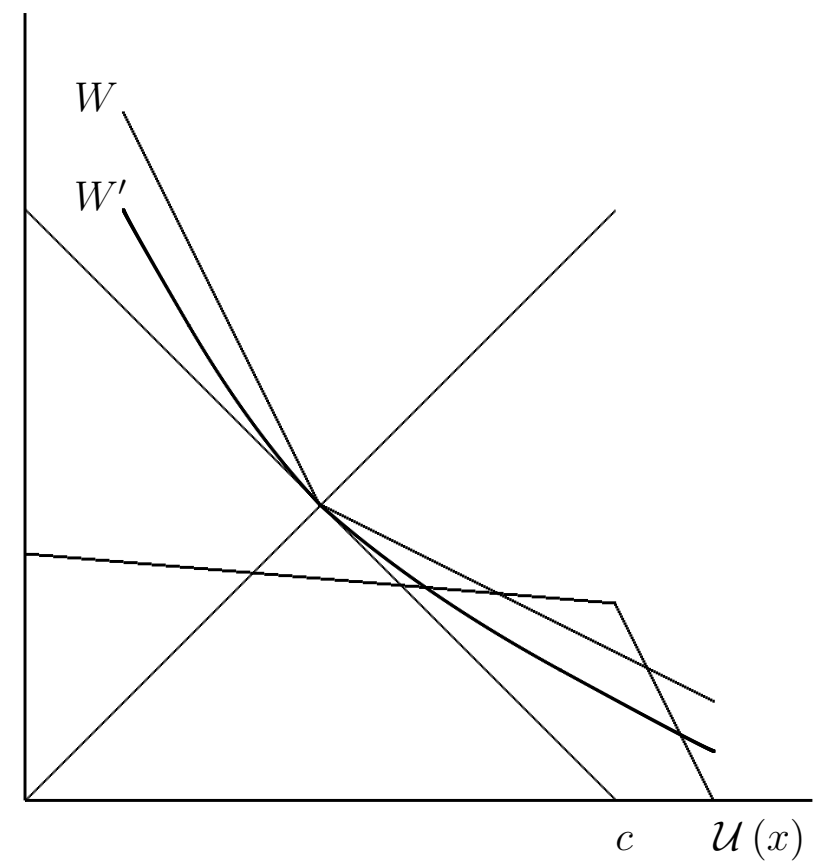

Figure 1: Proof of main result

an allocation for which for all $i \in N$ and all $s, s^{\prime}, x_{s}^{i}=x_{s^{\prime}}^{i}$ (that is; the allocation is independent of the state, featuring no uncertainty). As $\pi$ is a prior,

$$
U_{i}\left(x_{1}^{i}, \ldots, x_{\omega}^{i}\right) \leq\left(\pi_{1}, \ldots, \pi_{\omega}\right) \cdot\left(x_{1}^{i}, \ldots, x_{\omega}^{i}\right) .
$$

Consequently

$$
\begin{aligned}
\sum_{i=1}^{n} U_{i}\left(x_{1}^{i}, \ldots, x_{\omega}^{i}\right) & \leq \sum_{i=1}^{n}\left(\pi_{1}, \ldots, \pi_{\omega}\right) \cdot\left(x_{1}^{i}, \ldots, x_{\omega}^{i}\right) \\
& =\left(\pi_{1}, \ldots, \pi_{\omega}\right) \cdot(c, \ldots, c) \\
& =c .
\end{aligned}
$$

Now, let $\left\{\left(d^{1}, \ldots, d^{1}\right), \ldots,\left(d^{n}, \ldots, d^{n}\right)\right\}$ be an allocation of $c$ such that for all $i \in N$,

$$
U_{i}\left(x_{1}^{i}, \ldots, x_{\omega}^{i}\right) \leq d^{i} .
$$

As for any constant allocations $\left\{\left(d^{1}, \ldots, d^{1}\right), \ldots,\left(d^{n}, \ldots, d^{n}\right)\right\}$ of $c, U_{i}\left(d^{i}, \ldots, d^{i}\right)=d^{i}$, as both $W$ and $W^{\prime}$ are monotonic, it follows that they have maximizers on

$$
\left\{\left(d^{1}, \ldots, d^{n}\right): \sum_{i=1}^{n} d^{i}=c\right\} \text {. }
$$

As they are anonymous and quasiconcave, $\left(\frac{c}{n}, \ldots, \frac{c}{n}\right)$ maximizes each of them; these utilities are given by the allocation $\left\{\left(\frac{c}{n}, \ldots, \frac{c}{n}\right), \ldots,\left(\frac{c}{n}, \ldots, \frac{c}{n}\right)\right\}$. Now, suppose that 
$U_{W}\left(x_{1}, \ldots, x_{\omega}\right) \geq U_{W}(c, \ldots, c)$. Let $\left\{\left(\bar{x}_{1}^{1}, \ldots, \bar{x}_{\omega}^{1}\right), \ldots,\left(\bar{x}_{1}^{n}, \ldots, \bar{x}_{\omega}^{n}\right)\right\}$ solve

$$
\max _{\sum_{i=1}^{n} x_{s}^{i}=x_{s}} W\left(U_{1}\left(x_{1}^{1}, \ldots, x_{\omega}^{1}\right), \ldots, U_{n}\left(x_{1}^{n}, \ldots, x_{\omega}^{n}\right)\right) .
$$

Then

$$
W\left(U_{1}\left(\bar{x}_{1}^{1}, \ldots, \bar{x}_{\omega}^{1}\right), \ldots, U_{n}\left(\bar{x}_{1}^{n}, \ldots, \bar{x}_{\omega}^{n}\right)\right) \geq W\left(\frac{c}{n}, \ldots, \frac{c}{n}\right) .
$$

Consequently, as $W$ is more inequality averse than $W^{\prime}$,

$$
W^{\prime}\left(U_{1}\left(\bar{x}_{1}^{1}, \ldots, \bar{x}_{\omega}^{1}\right), \ldots, U_{n}\left(\bar{x}_{1}^{n}, \ldots, \bar{x}_{\omega}^{n}\right)\right) \geq W^{\prime}\left(\frac{c}{n}, \ldots, \frac{c}{n}\right) .
$$

Therefore,

$$
\begin{aligned}
& \max _{\sum_{i=1}^{n} x_{s}^{i}=x_{s}} W^{\prime}\left(U_{1}\left(x_{1}^{1}, \ldots, x_{\omega}^{1}\right), \ldots, U_{n}\left(x_{1}^{n}, \ldots, x_{\omega}^{n}\right)\right) \\
\geq & W^{\prime}\left(\frac{c}{n}, \ldots, \frac{c}{n}\right) \\
= & \max _{\sum_{i=1}^{n} x_{s}^{i}=c} W^{\prime}\left(U_{1}\left(x_{1}^{1}, \ldots, x_{\omega}^{1}\right), \ldots, U_{n}\left(x_{1}^{n}, \ldots, x_{\omega}^{n}\right)\right) .
\end{aligned}
$$

Consequently $U_{W^{\prime}}\left(x_{1}, \ldots, x_{\omega}\right) \geq U_{W^{\prime}}(c, \ldots, c)$.

\section{Examples}

Recall the example in Section 2. There, the maximax rule induced a more risk averse household utility than the utilitarian rule. The maximax rule is less inequality averse than the utilitarian rule; yet they are both anonymous. The reason for the violation of the theorem is that the maximax rule is itself not absolutely inequality averse.

The result also fails badly if the social choice functions under consideration are not anonymous. Again, consider two agents Alice and Bob. To discuss this example, we need not discuss the underlying states or preferences of the agents. Consider the two social welfare functions $W\left(u_{1}, u_{2}\right)=\inf \left\{u_{1}+3 u_{2}, \frac{7}{5}\left(u_{1}+2 u_{2}\right)\right\}$ and $W^{\prime}\left(u_{1}, u_{2}\right)=u_{1}+3 u_{2}$. Note that $W$ is more inequality averse than $W^{\prime}$. We claim that, in general, $W$ leads to a less risk averse household utility than $W^{\prime}$. To see why, fix some aggregate $\left(x_{1}, x_{2}\right)$. The utility possibility set for this aggregate bundle is given by

$$
\mathcal{U}\left(x_{1}, x_{2}\right)=\left\{\left(U_{1}\left(x_{1}^{1}, x_{2}^{1}\right), U_{2}\left(x_{1}^{2}, x_{2}^{2}\right)\right): x_{1}^{1}+x_{1}^{2}=x_{1}, x_{2}^{1}+x_{2}^{2}=x_{2}\right\} .
$$

With this notation, $U_{W}\left(x_{1}, x_{2}\right)=\max _{\left(u_{1}, u_{2}\right) \in \mathcal{U}\left(x_{1}, x_{2}\right)} W\left(u_{1}, u_{2}\right)$. Now, suppose that $U_{W^{\prime}}\left(x_{1}, x_{2}\right) \geq U_{W^{\prime}}(c, c)$. Recall that $\mathcal{U}(c, c)=\{(d, e): d+e=c\}$. Note that the maximizer of $W^{\prime}$ and of $W$ on $\mathcal{U}(c, c)$ is $(0, c)$. Therefore, $U_{W^{\prime}}\left(x_{1}, x_{2}\right) \geq U_{W^{\prime}}(c, c)$ implies that $\max _{\left(u_{1}, u_{2}\right) \in \mathcal{U}\left(x_{1}, x_{2}\right)} u_{1}+3 u_{2} \geq 3 c$. But note that $U_{W}(c, c)=\frac{14}{5} c$; consequently, as $u_{1}+3 u_{2} \geq 3 c$ implies both $\frac{7}{5}\left(u_{1}+2 u_{2}\right) \geq \frac{14}{5} c$ and $u_{1}+3 u_{2} \geq \frac{14}{5} c$, we conclude $\max _{\left(u_{1}, u_{2}\right) \in \mathcal{U}\left(x_{1}, x_{2}\right)}$ inf $\left\{u_{1}+3 u_{2}, \frac{7}{5}\left(u_{1}+2 u_{2}\right)\right\} \geq \frac{14}{5} c$, so that $U_{W}\left(x_{1}, x_{2}\right) \geq U_{W}(c, c)$. 


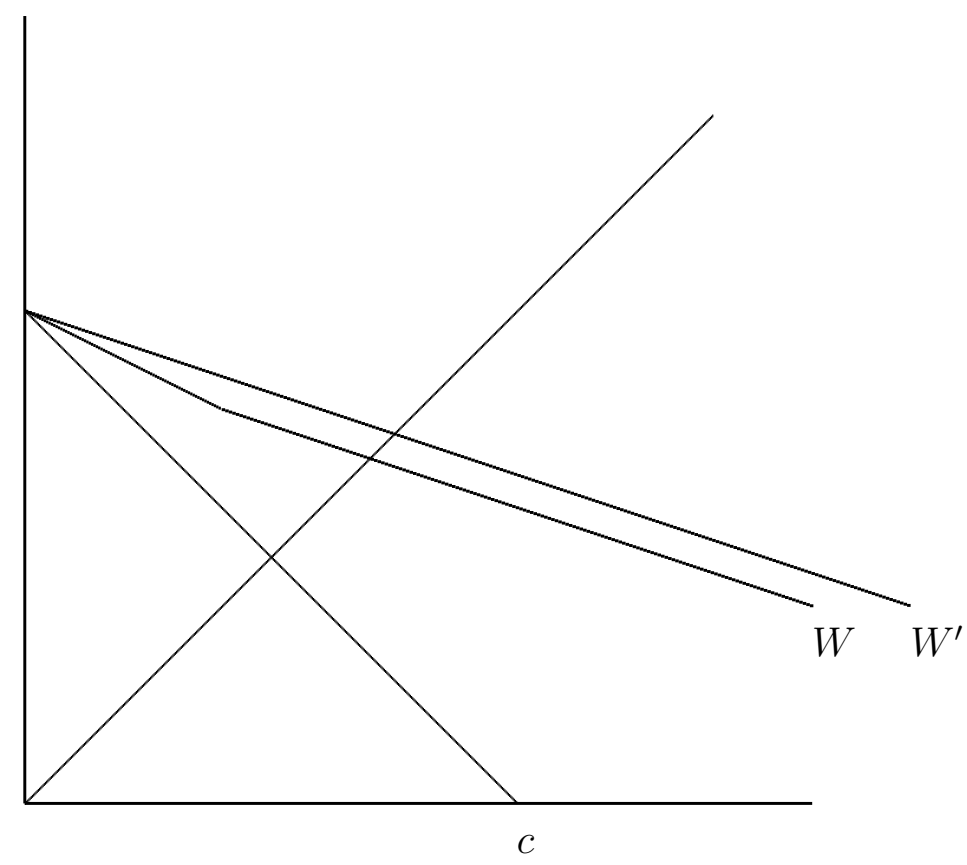

Figure 2: Non anonymous social welfare functions

Figure 2 illustrates the example. Each of $W$ and $W^{\prime}$ are maximized on the $c$-simplex at the same point. The level sets of $W$ and $W^{\prime}$ are each homothetic expansions of the level sets depicted. Note that, at the ray of equal coordinates, the at least as good as set of $W$ is always properly contained in that of $W^{\prime}$; but at the point $(0, c)$, the opposite is true. Therefore, $U_{W^{\prime}}$ is more risk averse than $U_{W}$.

\section{Conclusion}

The results of this note indicate that oftentimes, a household whose allocation decisions are motivated by equity will exhibit more risk averse behavior than one whose are not. This illuminates a basic tradeoff between household equity and aggregate risk attitudes.

While we have stated the result on the domain of inequality averse and anonymous social welfare functions, the proof makes clear that the critical property of these social welfare functions is their recommendation to equitably divide any dollar. Any pair of social welfare functions who recommend an equal division of a certain dollar will satisfy the comparative static discussed here.

Finally, we note that a partial converse of our result is available. Suppose we are given two inequality averse and anonymous social welfare functions, $W$ and $W^{\prime}$. Suppose it is known that for every list of utility functions with a common prior, $U_{W}$ is more risk 
averse than $U_{W^{\prime}}$. Then it follows that $W$ is more inequality averse than $W^{\prime}{ }^{3}$

\section{References}

Atkinson, A. (1970): "On the measurement of inequality," Journal of Economic Theory, 2(3), 244-263.

Chipman, J., And J. C. Moore (1979): "On social welfare and the aggregation of preferences," Journal of Economic Theory, 21(1), 111-139.

Samuelson, P. (1956): "Social indifference curves," Quarterly Journal of Economics, $70(1), 1-22$.

YAARI, M. E. (1969): "Some remarks on measures of risk aversion and on their uses," Journal of Economic Theory, 1(3), 315-329.

\footnotetext{
${ }^{3}$ This can be verified by a simple duality argument. Consider any upper contour set of $W$, passing through the ray of equal coordinates at $(c / n, \ldots, c / n)$, for example. Consdider any positive direction $p \in \mathbb{R}_{++}^{\Omega}$. Now consider the tangent hyperplane in direction $p$ to the upper contour set of $W$. This hyperplane is the boundary of a half space which is the utility possibilities set for some profile of utilities which are homogeneous of degree one (such a profile is easy to construct) and some aggregate bundle $x$ which returns positive payoff only in one state. The household utitily which is so constructed will rank $x$ as indifferent to $c$ according to $U_{W}$. As a consequence of the comparative risk aversion hypothesis, $x$ will be at least as good as $c$ according to $U_{W^{\prime}}$. Therefore, the tangent to the upper contour set of $W^{\prime}$ in direction $p$ will be further back than the corresponding tangent hyperplane of $W$. So long as each upper contour set is convex, this verifies that the upper contour set of $W^{\prime}$ contains that of $W$, and hence $W$ is more inequality averse than that of $W^{\prime}$. Unlike Theorem 1, this argument does rely critically on the fact that each of $W$ and $W^{\prime}$ are inequality averse.
} 